\title{
SEARCH FOR EMISSION LINE GALAXIES TOWARDS NEARBY VOIDS
}

\author{
C.C. POPESCU ${ }^{1}$, U. HOPP ${ }^{2}$ AND H. ELSÄSSER ${ }^{1}$ \\ ${ }^{1}$ Max Planck Institut f. Astronomie, D-69117 Heidelberg, FRG \\ ${ }^{2}$ Universitätssternwarte München, D-81679 München, FRG
}

Recent redshift surveys of galaxies (e.g. the Center for Astrophysics Survey (CfA)), revealed that bright galaxies are distributed in sheet-like structures which surround large voids. Still under debate is the question whether all galaxies follow such a distribution or if less luminous galaxies are more equally distributed. Given the limitation of the actual surveys, the observed emptiness of the voids may be a result of observational bias.

Several studies of the spatial distribution of galaxies, especially of dwarf galaxies, were carried out to overcome some of these biases. Unfortunately the results were contradictory and no definitive conclusions were drawn. We therefore started a project to search for emission-line galaxies (ELG) towards nearby voids in order to study their large scale spatial distribution. We use for this purpose the IIIa-J objective prism plates from the Hamburg QSO Survey. The plates are digitized and an automatic procedure was applied to select the candidates. All the selected objects were observed with follow-up spectroscopy, by means of the $2.2 \mathrm{~m}$ and $3.5 \mathrm{~m}$ telescopes of the Calar Alto Observatory.

We have obtained a final sample of 203 objects, of which 196 are ELG, four are galaxies with absorption lines and three are QSOs. Almost half of our objects are newly discovered ones and three quarters of the given redshifts are new. The apparent magnitudes, as derived from the objective prism plates, range between $15.0 \leq B \leq 19.5$. The sample is dominated by nearby galaxies, with a peak in the redshift distribution at 0.015 .

The first results indicate that there are a few very isolated ELG. Most of these are distributed along the rim of the voids. 